\title{
1 Séquence des événements à la centrale de Fukushima Dai-ichi
}

On trouvera en annexe une description détaillée de la séquence des événements ${ }^{1}$ de Fukushima. II ne s'agit ici que de résumer les phases principales. Nous présentons ici une analyse préliminaire qui intègre les informations aujourd'hui disponibles, mais il subsiste des incertitudes.ll faudra sans doute encore plusieurs années pour comprendre tout ce qui s'est passé à Fukushima, pour distinguer par exemple de manière claire les conséquences propres au séisme de celles du tsunami, et analyser la séquence des événements au sein des diverses parties de la centrale. Au fil des mois, des informations nouvelles seront disponibles et il serait opportun de revenir sur cette analyse, peut-être dans un cadre international.

Il faudra donc du temps pour déduire de cette analyse les erreurs qu'il ne faudrait pas répéter, ainsi que les mesures de sécurité que nous devrons prendre pour les réacteurs en service à l'heure présente. De plus, en juin 2011 , la situation à Fukushima reste fragile et à la merci d'une reprise sismique violente.

Néanmoins, on peut dire que, vraisemblablement, le séisme du 11 mars 2011 à 14 h 46, malgré sa magnitude 9, supérieure aux limites retenues pour la conception des réacteurs de la centrale de Fukushima ${ }^{2}$, n'aurait pas eu de conséquences très dommageables pour l'environnement ou la santé, s'il n'avait été suivi du tsunami. Il est possible que les tuyauteries reliant les vannes de dépressurisation des enceintes de confinement aux cheminées d'évacuation dans l'atmosphère aient pu être endommagées par le séisme. C'est peut-être cet endommagement qui a conduit aux explosions $d$ 'hydrogène dans les bâtiments réacteurs et donc à mettre en danger les piscines d'entreposage de combustible usé, par ailleurs non refroidies. L'analyse est encore incertaine et elle dépend d'investigations plus approfondies de l'état des réacteurs qui nécessiteront beaucoup de temps.

\footnotetext{
${ }^{1}$ Plusieurs sites permettent de suivre l'évolution de la situation des six réacteurs de la centrale de Fukushima Dai-ichi : notamment celui de l'autorité de sûreté japonaise NISA, http:// www.nisa.meti.go.jp/english/celui de l'Institut de radioprotection et de sûreté nucléaire, http:// www.irsn.fr/FR/Documents/home.htm celui de l'opérateur TEPCO, http://www.tepco.co.jp/en/ press/corp-com/release/index-e.html

On trouvera en annexe 1 un résumé de la séquence des événements initiaux.

${ }^{2}$ La construction avait été prévue pour résister à un séisme de magnitude 8 ; noter qu'un point en plus dans l'échelle de magnitude correspond à une énergie libérée trente fois supérieure. Néanmoins il est possible que l'accélération provoquée par le séisme au niveau du radier sur lequel repose un réacteur n'ait pas dépassé la valeur limite retenue par les concepteurs.
} 
Les dispositifs anti-sismiques ont fonctionné. Les réacteurs 1, 2 et 3 se sont mis à l'arrêt (les réacteurs 4,5 et 6 étaient déjà à l'arrêt pour maintenance), I'alimentation électrique par le réseau externe a été perdue, mais les générateurs électrogènes de secours nécessaires pour faire fonctionner les pompes permettant $d^{\prime}$ assurer le refroidissement des réacteurs et pour évacuer la puissance résiduelle se sont mis en marche. En effet, malgré l'arrêt des réacteurs, il reste un dégagement de chaleur considérable dû aux produits radioactifs qui se sont formés dans le combustible nucléaire pendant le fonctionnement du réacteur : plusieurs dizaines de mégawatts $(M W)$ dans les instants qui suivent l'arrêt $d^{\prime}$ 'un réacteur et encore une quinzaine de $\mathrm{MW}$ 24 heures plus tard. En l'état actuel des connaissances, il ne semble pas que les cuves des réacteurs aient été fissurées à la suite des secousses sismiques, ni les piscines dans lesquelles sont entreposés les assemblages de combustibles usés issus des réacteurs ${ }^{3}$, et il semble que les circuits hydrauliques nécessaires au refroidissement soient restés en état de fonctionner. Néanmoins, il sera sans doute difficile de caractériser l'état précis des installations juste après le séisme, avant l'arrivée du tsunami, compte tenu des endommagements qu'elles ont subis par la suite.

Les réacteurs étaient donc bien conçus pour répondre à un séisme et à une perte $d^{\prime}$ alimentation électrique externe. Mais ils n'étaient pas prêts à subir également pendant une période longue la perte des alimentations électriques de secours et de la source froide qu'a provoquée le tsunami. Celui-ci déferla 55 minutes après le séisme, noyant les générateurs électrogènes, des réacteurs 1-2-3 et $4^{4}$ et endommageant la prise d'eau de mer.

Pour chaque réacteur, il restait encore des systèmes de refroidissement de secours. Le refroidissement du cœur du réacteur 1 aurait dû être assuré par un système passif utilisant un réservoir d'eau situé au-dessus de la cuve (système nommé " Isolation Condenser ») et par un système d'injection d'eau à haute pression grâce à une pompe actionnée par une turbine utilisant la vapeur produite dans le cœur (système nommé « High-Pressure Coolant Injection system »). Le système passif qui s'était mis en route automatiquement avant la perte des générateurs de secours s'est arrêté de fonctionner et $n^{\prime}$ a pu être remis en service de manière continue ultérieurement. La turbo-pompe n'a pas démarré. Les causes précises de ces défaillances (erreur humaine, défaillance

\footnotetext{
${ }^{3}$ |l a subsisté une interrogation sur la piscine du réacteur $n^{\circ} 4$ qui contenait l'intégralité du combustible du réacteur à l'arrêt pour maintenance. Les combustibles se sontils trouvés à découvert par défaut de refroidissement et/ou une fuite survenue à la suite du séisme ? Un incendie s'est déclaré, dont l'origine est encore inconnue. Après reconnaissance par des robots, les assemblages de combustible usé sont intacts.

${ }^{4}$ Les réacteurs 5 et 6 ont été construits en 1978 et 1979 à une dizaine de mètres au-dessus du niveau des quatre premiers (1, 2 et 3 datent de 1970 à 1974) et leurs générateurs de refroidissement $n^{\prime}$ ont sans doute pas été noyés. Leur situation paraissait être maîtrisée à la date d'aujourd'hui (18/4/2011). Un seul des quatre diesels a fonctionné ce qui est suffisant.
} 
matérielle due par exemple à la défaillance des batteries de secours) ne sont pas connues précisément. II semble donc qu'il n'y ait eu aucun refroidissement du cœur du réacteur 1 pendant une durée de 14 heures environ.

Pour les réacteurs 2 et 3 , le refroidissement a pu être momentanément assuré par des pompes actionnées par des turbines utilisant la vapeur produite dans le cœur (systèmes nommés « Reactor Core Isolation Cooling system »). Ces pompes ont permis d'injecter de l'eau froide depuis des réservoirs et de faire circuler l'eau contenue dans les tores annulaires situés en partie inférieure des bâtiments. Le système d'injection d'eau à haute pression a également démarré automatiquement sur le réacteur 3. Mais tous ces moyens ont été perdus pour diverses raisons qui restent encore à éclaircir (défaillance des batteries, indication de niveau d'eau haut dans la cuve, chute de pression dans la cuve). Il semble donc qu'il n'y ait également eu aucun refroidissement des cœurs des réacteurs 2 et 3 pendant une durée de 7 heures environ.

Il faut noter qu'en l'absence de restauration de la source froide, ces moyens de secours n'auraient pu permettre de refroidir de manière pérenne les cœurs des réacteurs. Une injection d'eau froide par un moyen complémentaire était nécessaire, ce qui a pu être finalement réalisé, mais bien trop tardivement.

En l'absence de refroidissement, la chaleur dégagée par la radioactivité du combustible vaporise progressivement l'eau de la cuve, puis chauffe la vapeur; la pression à l'intérieur de la cuve augmente. Lorsque la température dépasse $800-900^{\circ} \mathrm{C}$, la réaction d'oxydation par la vapeur d'eau des gaines métalliques en alliage de zirconium qui recouvrent le combustible ${ }^{5} s^{\prime}$ accélère fortement, libérant de grandes quantités d'hydrogène et d'énergie car la réaction est très exothermique ${ }^{6}$. Cela se produit vraisemblablement en moins d'une heure.

Dans ces périodes d'assèchement, le combustible cesse progressivement de baigner dans l'eau liquide; vers les $900^{\circ} \mathrm{C}$, les structures des barres de contrôle (carbure de bore dans une gaine en acier dans les réacteurs à eau bouillante) se liquéfient, puis à $1800^{\circ} \mathrm{C}$ les gaines des crayons de combustible let les boîtiers des assemblages dans le cas des réacteurs à eau bouillante) en Zircaloy fondent; à partir de $2300^{\circ} \mathrm{C}$ le combustible commence à se liquéfier, notamment par suite de sa dissolution par les matériaux de structure fondus et il se forme un magma mélangeant les matériaux fondus, le « corium », à très haute température.

\footnotetext{
${ }^{5}$ Elles sont en zirconium, métal transparent aux neutrons qui, à $1200{ }^{\circ} \mathrm{C}$ en présence de vapeur d'eau, réduit l'eau en formant de l'oxyde de zirconium et de l'hydrogène.

${ }^{6}$ Les enthalpies libres des réactions d'oxydation de $\mathrm{Zr}$ par $\mathrm{H}_{2} \mathrm{O}$ et $\mathrm{O}_{2}$ sont respectivement de $-459 \mathrm{~kJ} / \mathrm{mol} \mathrm{Zr}$ et $-755 \mathrm{~kJ} / \mathrm{mol} \mathrm{Zr}$.
} 
Pour diminuer la pression interne ${ }^{7}$ de la cuve du réacteur et de l'enceinte de confinement, les opérateurs ont fait des relâchements de vapeur, mais, au contact de l'air dans les bâtiments, l'hydrogène ainsi dégagé avec la vapeur a explosé, emportant les couvertures métalliques des bâtiments 1 puis 3 , avec des dommages encore plus importants sur ce dernier réacteur. Notons que le réacteur $n^{\circ} 3$ utilisait du combustible $M O X^{8}$ en faible proportion, mais cela $n^{\prime} a$ probablement pas eu $d^{\prime}$ incidence sur la nature des rejets de matières radioactives, les composés formés à partir des transuraniens étant très peu volatils.

La reprise de l'alimentation en eau, par injection d'eau de mer par le réseau de protection contre les incendies, a réussi à arrêter le processus d'échauffement des réacteurs. Mais, à partir du 17 mars, une inquiétude nouvelle s'est ajoutée concernant les piscines d'entreposage des assemblages de combustible, notamment celle du réacteur $n^{\circ} 4$; la question s'est posée de savoir si ceux-ci pouvaient se retrouver découverts au contact de l'air lla chaleur dégagée par la radioactivité des combustibles suffit, en cas d'arrêt du refroidissement à mettre à découvert le combustible entreposé dans une piscine en une à plusieurs dizaines de jours) selon le nombre ef l'activité des assemblages de combustible usé entreposés. Le séisme a-t-il projeté une grande quantité d'eau hors de la piscine, ou celle-ci s'est-elle fissurée, nous ne connaissons pas encore la réponse. Le danger potentiel est donc là considérable puisque l'on pourrait avoir au plan de la radioactivité l'équivalent d'une fusion de cœur à l'air libre 9 , sans aucun confinement des produits de fission libérés, car il n'y avait pas d'enceinte de confinement prévue pour ces piscines $^{10}$. Les assemblages de combustible usé entreposés $n^{\prime}$ ont a priori pas été endommagés, comme le confirment les mesures de radioactivité qui ont été effectuées autour du site ${ }^{11}$ et les reconnaissances réalisées à l'aide de robots.

\footnotetext{
${ }^{7}$ Montée dans le tore, en principe séparé de l'enceinte de confinement à 8 ou 9 bars semble$\mathrm{t}$ - il sur le réacteur $n^{\circ} 1$, alors que la résistance nominale n'était garantie que jusqu'à 5 bars. La décharge de la vapeur de l'enceinte du réacteur se fait à travers l'eau de la piscine du tore qui retient une partie de la radioactivité, mais cela peut enrichir la vapeur en hydrogène par condensation d'eau. La décharge de la vapeur de l'enceinte de confinement se fait ensuite, en principe, à la cheminée. Une explosion très violente a eu lieu au niveau du tore du réacteur 2 avec fuite d'eau radioactive dans le bâtiment des turbines.

${ }^{8}$ MOX: Mixed Oxide Fuel composé du plutonium issu du retraitement pour $7 \%$ et de I'uranium appauvri ${ }^{238} \mathrm{U}$, résidu des opérations d'enrichissement, pour $93 \% .32$ assemblages sur les 548 présents dans le cœur étaient composés de crayons de combustible MOX.

${ }^{9}$ À la différence importante près qu'il n'y aurait probablement presque plus eu de dégagement d'iode 131, mais il y avait 1300 assemblages de combustible usé du réacteur 4 , soit plusieurs cœurs.

${ }^{10}$ Hormis le bâti métallique externe. L'explosion de ces enceintes pour les réacteurs 1 et 3 a sans doute permis de réalimenter par les lances à incendie les piscines des combustibles usés de ces réacteurs.

${ }^{11}$ Il est estimé notamment que les rejets en césium auraient été alors supérieurs d'un ordre de grandeur à ceux ayant résulté des rejets des réacteurs accidentés, induisant des conséquences radiologiques bien supérieures à celles observées.
} 
Le 13 avril, TEPCO annonçait une température de l'eau de cette piscine qui était encore de $90^{\circ} \mathrm{C}$, supérieure aux $40^{\circ} \mathrm{C}$ normaux, mais en dessous de la température d'ébullition de l'eau.

Au 25 août 2011 , il semble que la situation soit en voie d'être maîtrisée (en l'absence de nouveau séisme, mais la terre continue à trembler) et qu'il n'y ait plus que de faibles émissions de produits radioactifs. Les réacteurs 1, 2 et 3 sont maintenant refroidis par injection d'eau douce directement dans les cuves (débit d'environ $\left.15 \mathrm{~m}^{3} / \mathrm{h}\right)^{12}$. L'eau qui s'en échappe, fortement contaminée, est prélevée depuis les bâtiments des turbines, traitée dans trois installations mises en service à partir du mois de juin puis, après désalinisation, réinjectée dans les cuves. De plus, une injection d'azote est effectuée dans les trois enceintes de confinement pour les maintenir inertes et ainsi éviter tout risque de combustion d'hydrogène.

Mais la maîtrise ne sera réellement complète que lorsque les cœurs seront refroidis en circuit fermé. Les mesures de la radioactivité émise par cet accident majeur, classé au niveau 7 de l'échelle $\mathrm{INES}^{13}$, niveau le plus élevé, indiquent des rejets dans l'atmosphère environ dix fois inférieurs à ceux de Tchernobyl. Mais cette émission à beaucoup moins haute altitude a conduit à proximité de la centrale dans une région très peuplée à des dépôts radioactifs comparables à ceux de Tchernobyl. L'évacuation de la zone des $20 \mathrm{~km}$ autour de la centrale (c'est-à-dire d'environ 170000 habitants), intervenue avant les relâchements majeurs de radioactivité, a sans doute réduit l'impact de celle-ci mais il n'y a pas encore d'estimation précise des doses et débits de doses auxquels les habitants ont été soumis.

Par ailleurs, par suite de conditions météorologiques particulièrement défavorables lors des rejets ayant eu lieu du 15 au 16 mars (vent soufflant vers I'intérieur des terres, conjugué à des précipitations abondantes sous forme de pluies et de chutes de neige), une bande de territoire d'environ $20 \mathrm{~km}$ de large et $60 \mathrm{~km}$ de long située au nord-ovest du site a été soumise à des dépôts d'iode et de césium importants. L'évacuation des 70000 habitants y résidant a été décidée par les autorités japonaises deux mois après l'accident afin de réduire l'exposition au césium 137 qui subsiste. L'existence de cette zone constitue avec la zone d'exclusion initiale des $20 \mathrm{~km}$ sans doute l'élément le plus grave provoqué par cette catastrophe.

On imagine parfois qu'un réacteur non contrôlé pourrait engendrer une explosion atomique. II faut tout de même bien préciser qu'il n'en est rien et

${ }^{12}$ Les premiers calculs du déroulement de l'accident indiquent que la cuve du réacteur $n^{\circ} 1$ a dû être percée par le corium en fusion en libérant une partie qui se serait répandue sur le radier en béton du réacteur.

${ }^{13}$ International Nuclear Event Scale. 
qu'un scénario apocalyptique de cette nature est heureusement exclu. Les accidents nucléaires passés, même les plus graves, sont dus à des augmentations de température et de pression «classiques » et non à une réaction en chaîne explosive. Le combustible d'un réacteur, uranium enrichi à moins de $5 \%$ en isotope 235, ainsi que le plutonium produit par le fonctionnement du réacteur, ne permettent absolument pas, quelle que soit la séquence des événements, d'engendrer une explosion nucléaire ${ }^{14}$.

L'explosion de Tchernobyl était due à une brusque augmentation de pression de l'eau provoquée par l'emballement de la puissance du réacteur. À Fukushima, les explosions ont été dues à des fuites d'hydrogène, les réactions de fission ayant été arrêtées dès les premières secousses du séisme.

Le refroidissement de fortune des réacteurs et des piscines à conduit à $d^{\prime}$ importants volumes d'eau contaminée dont le traitement vient de commencer selon un procédé indiqué en annexe. La décontamination des sols et la gestion des déchets interviendront ultérieurement.

\section{Quelques interrogations complémentaires}

- Les réacteurs 1 à 4 ont été implantés en creusant la falaise côtière qui est à environ $40 \mathrm{~m}$ au-dessus du niveau de la mer. Peutêtre s'agissait-il de trouver un soubassement rocheux plus stable en cas de séisme, ou alors simplement de faciliter le pompage? En tout cas, l'exposition au tsunami n'aurait pas eu lieu si la centrale avait été construite sur la falaise. Visiblement les constructeurs ont pris conscience de ce danger en implantant les réacteurs 5 et 6 une dizaine de mètres plus haut ${ }^{15}$.

- On mesure à quel point il est essentiel de prémunir les générateurs diesel de secours contre une inondation.

- La déficience des systèmes de secours pour le contrôle commande des turbines situées dans le réacteur a été un facteur d'aggravation important. En effet, ceux-ci sont susceptibles tant qu'ils fonctionnent de maintenir sous contrôle le réacteur en cas de perte simultanée de I'alimentation électrique externe et des sources de refroidissement. On ne peut que s'interroger sur les causes de cette panne (intervenue sur le réacteur $n^{\circ} 1$ au bout de deux heures). Néanmoins, en l'absence de la source froide, perdue à cause du tsunami, le refroidissement $n^{\prime}$ aurait pu être assuré très longtemps car l'eau des tores serait de toute façon entrée en ébullition en entraînant la perte de ces systèmes.

${ }^{14}$ Pour fabriquer une bombe, il faut de l'uranium 235 presque pur, en tout cas enrichi à au moins $80 \%$. La dispersion de $\mathrm{I}^{\prime 235} \mathrm{U}$ dans $\mathrm{I}^{\prime 238} \mathrm{U}$ dans un réacteur interdit toute «explosion atomique ».

15 Les réacteurs 5 et 6 sont en « arrêt froid », situation maîtrisée, depuis le 20 mars. 
- L'absence ou l'inefficacité d'installation de récupération et de recombinaison d'hydrogène dans les bâtiments situés au-dessus de l'enceinte de confinement des réacteurs a conduit aux explosions qui se sont produites au-dessus des réacteurs 1 et 3 . Dans le réacteur 2 , il s'est agi d'une explosion dans le tore. Notons ici que des recombineurs d'hydrogène autocatalytiques passifs (RAP) ont été installés fin 2007 sur tous les réacteurs en service en France (voir plus bas).

- L'exploitant TEPCO a t-il disposé des moyens d'intervention adaptés aux opérations de secours dans des locaux irradiants? De même, on peut $s^{\prime}$ interroger sur le délai assez long qui a été nécessaire pour acheminer et mettre en opération des camions-pompes ayant la puissance nécessaire. Certes, les dégâts subis par les voies de communication ainsi que les délicates décisions d'affectation des moyens de secours ont dû jover un rôle, mais une analyse approfondie des raisons de ces délais s'impose. Il serait également intéressant de savoir si les robots du groupement français INTRA ${ }^{16}$ auraient été capables $d^{\prime}$ intervenir efficacement dans ce cas.

- L'absence d'enceinte de confinement au-dessus des piscines de combustible peut conduire, en cas de perte de refroidissement pendant plusieurs jours, à un danger gravissime, parce qu'à l'heure actuelle rien n'est prévu pour limiter les rejets radioactifs que cela pourrait entraîner ${ }^{17}$. On voit combien il est important de limiter autant que possible le nombre d'assemblages de combustible usé entreposés dans les piscines situées dans les bâtiments des réacteurs.

\section{2 | La situation du nucléaire en France après Fukushima}

L'accident de Fukushima a montré qu'un événement hautement improbable, tel une perte simultanée et sur des temps longs des alimentations électriques et des sources froides conduisant à endommager gravement trois réacteurs électronucléaires d'une centrale, est néanmoins arrivé. Il faut donc réévaluer le niveau de sûreté de nos installations nucléaires sans écarter comme impossibles des événements de très faible probabilité et sans négliger la possibilité d'occurrence simultanée de plusieurs événements rares a priori indépendants.

${ }^{16}$ Groupe d'intervention robotique sur accident, créé en 1988 par EDF-CEA-COGEMA.

17 Dans les EPR, la piscine d'entreposage est située à l'intérieur de la coque « avion » offrant ainsi une plus grande robustesse vis-à-vis d'un impact important. Mais, pour autant, cette coque ne joue aucun rôle de confinement des produits radioactifs. 